\title{
Hinweise für die Benutzung
}

1. Behandelt wird die Zwangsvollstreckung wegen Geldforderungen, soweit sie in das bewegliche Vermögen und nach der Zivilprozessordnung betrieben wird. Für die Vollstreckung wegen Steuerforderungen gilt die Abgabenordnung. Die Muster sind auch für die Vollstreckung wegen Steuerforderungen und auch wegen nach den Verwaltungsvollstreckungsgesetzen beizutreibender Geldforderungen (vgl. $\$ 4$ $\mathrm{VwVG}$ ) verwendbar.

2. Der erste Teil des Buchs, „Einleitung“ genannt (A.), gibt einen Überblick über das Vollstreckungsverfahren nach der Zivilprozessordnung und zeigt die beiden zentralen, verbindlichen Formulare (B.), s. auch unter 4.

3. Der zweite Teil des Buchs enthält Musteranträge zur Sach- und Forderungspfändung.

Die Muster 1 bis 11 (C.) zeigen Anträge, die regelmäßig der Forderungspfändung vorausgehen und solche, die der Sachpfändung dienen, geordnet nach üblicher zeitlicher Reihenfolge.

Alle weiteren Muster (D.) enthalten Formulierungsvorschläge für die Pfändung und Verwertung von Forderungen und anderen Rechten; die Überschriften (Stichwörter) sind alphabetisch geordnet. Finden sich zu einem Stichwort mehrere Muster, so helfen Untertitel bei der Auswahl.

4. Verbindliche Formulare: $\mathrm{Zu}$ beachten sind die Formulare für die Pfändung wegen gewöhnlicher Forderungen und wegen Unterhaltsforderungen (B.) aufgrund der Verordnung über Formulare für die Zwangsvollstreckung (Zwangsvollstreckungsformular-Verordnung - ZVFV) vom 23.8.2012 (BGBl. I 2012, S. 1822), geändert aufgrund Verordnung zur Änderung der Zwangsvollstreckungsformular-Verordnung vom 16.6.2014 (BGBl. I 2014, S. 754).

Ein ebenfalls zwingend zu nutzendes Formular hat das BMJV für den Antrag auf Erlass einer richterlichen Durchsuchungsanordnung (Muster M 11) erlassen (Anlage 1 zur ZVFV).

Das BMJV hat auch von der Ermächtigung des $₫ 753$ Abs. 3 ZPO Gebrauch gemacht und nunmehr auch ein Formular für den Vollstreckungsauftrag an den Gerichtsvollzieher (Gerichtsvollzieherformular-Verordnung - GVFV) eingeführt. Mit Wirkung vom 1.12.2016 ist das Formular insbesondere wegen neuer rechtlicher Regelungen überarbeitet worden. Die neuen Regelungen sind mit dem Gesetz zur Durchführung der Verordnung (EU) Nr. 655/2014 sowie zur Änderung sonstiger zivilprozessualer, grundbuchrechtlicher und vermögensrechtlicher Vorschriften und zur Änderung der Justizbeitreibungsordnung (EuKoPfVODG) vom 21.11.2016 (BGBl. I 2016, S. 2591) eingeführt worden (Muster M 2).

Für den Erlass des Pfändungs- und Überweisungsbeschlusses ist allerdings nur der Antrag verbindlich vorgegeben. Der Inhalt des gerichtlichen Beschlusses selbst kann jedoch nicht vom Verordnungsgeber verbindlich vorgeschrieben werden. Und soweit die Forderung durch einen Beschluss bereits gepfändet worden ist, ist für den 
Antrag auf Überweisung dieser Forderung die Nutzung der Formulare ebenfalls nicht verbindlich.

Kritik: Wenn der Verordnungsgeber nach $\$ 3$ ZVFV a.F., $\$ 5$ ZVFV n.F. die Formulare ab dem 1.3.2013 bzw. ab dem 1.11.2014 für verbindlich erklärt, kann dies so ausgelegt werden, dass für den Antragsteller nicht nur der Antrag, sondern das gesamte Formular (= Formularfelder) verbindlich auszufüllen ist. Dies korrespondiert mit dem Gesetzestext in $\$ 829$ Abs. 4 Satz 2 ZPO, der Antragsteller muss die verbindlichen Formulare benutzen und wohl auch die neuen Texten nach der Änderungs-VO. Dies ändert aber nichts an der Tatsache, dass die weiteren Formularfelder nach dem Antrag nur als „Entwurf“ für den zu erlassenden Pfändungs- und Überweisungsbeschluss dienen. Das Vollstreckungsgericht ist an die ausgefüllten Formularfelder textlich nicht gebunden. Nach Herget (Zöller/Herget, \$829 ZPO Rz. 43) sind daher für den Antragsteller die Formularfelder im Entwurf für den Pfändungsund Überweisungsbeschluss ebenfalls nicht verbindlich. Allerdings sind nach $\$ 3$ Abs. 1 Satz 1 ZVFV inhaltliche Abweichungen von den Formularen unzulässig. Soweit für den beabsichtigten Antrag keine zweckmäßige Eintragungsmöglichkeit in dem Formular besteht, kann ein geeignetes Freifeld oder eine Anlage genutzt werden. Die Nutzung mehrerer Freifelder und Anlagen ist zulässig. Es reicht aus, wenn der Antragsteller nur die Seiten des Formulars, auf denen sich Angaben des Antragstellers befinden, bei dem Gericht einreicht. Die nicht eingereichten Formularseiten sind auch in diesem Fall Teil des Antrags.

Mehrfach hat sich der BGH mit den Formularen befassen müssen. Mit Beschluss v. 13.2.2014 - VII ZB 39/13 (Rpfleger 2014, 272 = WM 2014, 512) entschied der BGH wie folgt:

a) Die den Formularzwang für Anträge auf Erlass eines Pfändungs- und Überweisungsbeschlusses regelnden Rechtsnormen können verfassungskonform dahingehend ausgelegt werden, dass der Gläubiger vom Formularzwang entbunden ist, soweit das Formular unvollständig, unzutreffend, fehlerhaft oder missverständlich ist.

b) In diesen, seinen Fall nicht zutreffend erfassenden Bereichen ist es nicht zu beanstanden, wenn er in dem Formular Streichungen, Berichtigungen oder Ergänzungen vornimmt oder das Formular insoweit nicht nutzt, sondern auf beigefügte Anlagen verweist.

c) Ein Antrag auf Erlass eines Pfändungs- und Überweisungsbeschlusses ist nicht formunwirksam, wenn sich der Antragsteller eines Antragsformulars bedient, das im Layout geringe, für die zügige Bearbeitung des Antrags nicht ins Gewicht fallende Änderungen enthält.

d) Ein Antrag auf Erlass eines Pfändungs- und Überweisungsbeschlusses ist auch nicht deshalb formunwirksam, weil das Antragsformular nicht die in dem Formular gemäß Anlage 2 zu $\$ 2$ Nr. 2 ZVFV enthaltenen grünfarbigen Elemente aufweist.

In den Gründen des Beschlusses heißt es u.a.:

„... Gemessen hieran würde der allgemeine Justizgewährungsanspruch in verfassungswidriger Weise eingeschränkt, wenn der gesetzlich geregelte Formularzwang so zu verstehen wäre, dass die Formulare ohne Einschränkung zu verwenden wären. Mit dem Formularzwang wird insbesondere eine Entlastung der Vollstreckungsgerichte bezweckt. Durch die Vereinheitlichung der Antragsformulare soll die Effizienz bei der Bearbeitung der Anträge bei Gericht gesteigert werden (vgl. BT-Drucks. 13/341, S. 11; BR-Drucks. 326/12, S. 1). Die Umsetzung dieses in Anbetracht der Vielschichtigkeit der Forderungspfändung anspruchsvollen gesetz- 
geberischen Anliegens durch die verbindliche Vorgabe des Formulars gemäß Anlage 2 zu $\$ 2$ Nr. 2 ZVFV schränkt den Anspruch des Rechtsuchenden auf effektiven Rechtsschutz unverhältnismäßig ein. Das vorgegebene, verbindlich zu nutzende Formular ist an mehreren Stellen unvollständig und zum Teil widersprüchlich sowie missverständlich. Zudem weist es in Teilbereichen rechtliche Unzulänglichkeiten auf. ...

Beispielhaft sei ausgeführt:

aa) Forderungsaufstellung (Seite 3): Die Forderungsaufstellung auf Seite 3 zeigt sich für eine Vielzahl der praktischen Fälle als ungeeignet. Sie ist darüber hinaus in sich missverständlich.

So ist aufgrund des Aufbaus des Formulars, das in der ersten und vorletzten Zeile die Möglichkeit vorsieht, auf eine anliegende Forderungsaufstellung zu verweisen, unklar, ob zwingend in der ersten Spalte ein Betrag einzutragen ist oder ob alternativ dazu auf eine beigefügte Forderungsaufstellung verwiesen werden kann.

Missverständlich sind zudem die Zeilen 3 und 4 betreffend die Zinsansprüche. So kann das Formular dahingehend verstanden werden, dass in der ersten Spalte die ausgerechneten Zinsen einzutragen sind, die sodann in der zweiten Spalte erläutert werden, wofür der Gesamtaufbau der Forderungsaufstellung spricht. Bei einem solchen Verständnis des Formulars findet der Antragsteller jedoch keine Eintragungsmöglichkeit für die weiteren, ab Antragstellung laufenden Zinsen. Die Zeilen 3 und 4 können jedoch auch dahingehend aufgefasst werden, dass in die linke Spalte die ausgerechneten aufgelaufenen Zinsen und in die zweiten Spalte die weiteren Zinsen ab Antragstellung aufzunehmen sind.

Das Formular ist darüber hinaus in den Fällen, in denen wegen mehrerer Hauptforderungen die Vollstreckung betrieben wird, ungeeignet, da lediglich eine Hauptforderung in die Forderungsaufstellung eingetragen werden kann ... Ob der Gläubiger die Forderungsaufstellung dennoch (teilweise) nutzen muss und eventuell die Summe der Forderungen in die Summenzeile eintragen muss oder insgesamt auf eine Anlage verweisen kann, erschließt sich nicht.

Das Formular erlaubt zudem weder die Eintragung von Teilzahlungen des Schuldners noch von gestaffelten Zinsen. Für den Antragsteller ist in diesen Fällen nicht erkennbar, ob - und wenn ja welche - Beträge in die Forderungsaufstellung aufzunehmen sind und inwieweit auf eine Anlage verwiesen werden darf.

Darüber hinaus ist die Eintragung einer Vorsteuerabzugsberechtigung, was hinsichtlich der Vollstreckungskosten erforderlich sein kann, nicht vorgesehen. Auch insoweit bleibt unklar, an welcher Stelle eine entsprechende Eintragung vorzunehmen ist.

Das Formular eröffnet dem Antragsteller hinsichtlich der zu pfändenden Ansprüche keine genügende Auswahlmöglichkeit. Hat der Gläubiger auf Seite 4 in dem ersten Kasten „Forderung aus Anspruch“ einen oder mehrere Bereiche ausgewählt, so ist der Antrag unumgänglich auf Pfändung sämtlicher in dem Formular dem ausgewählten Drittschuldner zugeordneter Ansprüche gerichtet. Zudem besteht, wenn auf Seite 3 unten mehrere Drittschuldner eingetragen werden, keine Möglichkeit, die zu pfändenden Forderungen auf den Seiten 4 bis 6 sowie die Anordnungen auf den Seiten 7 und 8 den einzelnen Drittschuldnern zuzuordnen. Stets wird dadurch bei allen Drittschuldnern die Pfändung der gleichen Ansprüche und der Erlass der gleichen Anordnungen beantragt. Die Dispositionsbefugnis des Gläubigers wird durch diese verbindlichen Vorgaben des Formulars in rechtswidriger Weise eingeschränkt .... .

Darüber hinaus besteht auch keine Möglichkeit, die Felder „Anspruch C (an Finanzamt)“ bis „Anspruch G (an Sonstige)“ auf den Seiten 4 bis 6 sowie die Anordnungen auf den Seiten 7 und 8 auszublenden, soweit sie für den konkreten Antrag nicht von Relevanz sind. Der Antragsteller ist gehalten, stets das gesamte neunseitige Antragsformular bei dem Vollstre- 
ckungsgericht einzureichen, obschon mehrere Seiten für seinen konkreten Antrag ohne Bedeutung sein können.

\section{Nicht zuletzt im Hinblick auf die im Bereich der Forderungspfändung häufig bestehende Eilbedürftigkeit ist die uneingeschränkte verbindliche Nutzung des Formulars für den einzelnen Rechtsuchenden daher unzumutbar.}

Nach diesen Grundsätzen sind die den Formularzwang regelnden Normen verfassungsgemäß dahingehend auszulegen, dass der Gläubiger vom Formularzwang entbunden ist, soweit das Formular unvollständig, unzutreffend, fehlerhaft oder missverständlich ist. In diesen, seinen Fall nicht zutreffend erfassenden Bereichen ist es nicht zu beanstanden, wenn er in dem Formular Streichungen, Berichtigungen oder Ergänzungen vornimmt oder das Formular insoweit nicht nutzt, sondern auf beigefügte Anlagen verweist."

\section{Fazit:}

Nach dieser Entscheidung des BGH vom 13.2.2014 wartete die Praxis gespannt, wie das BMJ reagieren würde. Die geringfügigen Änderungen aufgrund der ÄnderungsVO vom 16.6.2014 zeigen, dass das BMJ so gut wie nicht reagiert hat. Die „SEPAUmstellung“ leuchtet ein, ebenfalls u.a. die Trennung in Pfändungs- und Überweisungsbeschluss, aber die Kernprobleme sind geblieben.

Noch deutlicher wurde der $\mathrm{BGH}^{1}$ in seinen Beschlüssen vom 20.2.2014 - VII ZB 44/13, vom 20.2.2014 - VII ZB 31/13 und vom 20.2.2014 - VII ZB 46/13. Im Kern wurde entschieden:

a) Die den Formularzwang für Anträge auf Erlass eines Pfändungs- und Überweisungsbeschlusses regelnden Normen sind nach ihrem Sinn und Zweck dahingehend auszulegen, dass auch die Nutzung solcher Formulare möglich ist, die im Layout geringe, für die zügige Bearbeitung des Antrags nicht ins Gewicht fallende Änderungen enthalten.

b) Der Gläubiger ist vom Formularzwang entbunden, soweit das Formular unvollständig, unzutreffend, fehlerhaft oder missverständlich ist.

c) In den Bereichen, in denen das Formular aus diesen Gründen den Fall des Gläubigers nicht zutreffend erfasst, ist es nicht zu beanstanden, wenn er dem Formular eine Anlage beifügt oder in dem Formular zusätzliche Eintragungen vornimmt, selbst wenn das Formular an dieser Stelle keine oder eine für die Eintragung zu geringe Anzahl an Freizeilen aufweist.

Weiter heißt es in zwei weiteren Beschlüssen des BGH vom 4.11.2015 - VII ZB 22/15 (Rpfleger 2016, 167) und vom 11.5.2016 - VII ZB 54/15 (Rpfleger 2016, 660):

Bietet das Antragsformular gemäß Anlage 2 zu $\$ 2$ Satz 1 Nr. 2 ZVFV hinsichtlich der Forderungsaufstellung eine vollständige Eintragungsmöglichkeit, ist ausschließlich das vorgegebene Formular zu nutzen.

1 Zur GVFV hat der BGH (v. 26.9.2018 - VII ZB 56/16, Rpfleger 2019, 46 = DGVZ 2018, 248) mittlerweile ähnlich entschieden: 1. Der Gläubiger ist nur vom Formularzwang gemäß $\S \$ 1,5$ GVFV entbunden, soweit das Formular unzutreffend, fehlerhaft oder missverständlich ist. 2. Für (sonstige) Hinweise, die die beabsichtigte Zwangsvollstreckung betreffen, ist das Modul P 8 des Formulars vorgesehen. Nicht titulierte Forderungen und Hinweise auf nicht titulierte Forderungen betreffen nicht die Zwangsvollstreckung und dürfen deshalb nicht in das Formular aufgenommen werden. 
Mit Beschluss vom 15.6.2016 - VII ZB 58/15 (Rpfleger 2016, 740) wurde der BGH nochmals mit der Frage zur Nutzung des Formulars konfrontiert:

a) Sofern das Antragsformular gemäß Anlage $2 \mathrm{zu} \S 2$ Satz 1 Nr. 2 ZVFV für den Antrag des Gläubigers auf Erlass eines Pfändungs- und Überweisungsbeschlusses hinsichtlich der zu vollstreckenden Forderungen auf Seite 3 keine vollständige und zutreffende Eintragungsmöglichkeit bietet, ist es nicht zu beanstanden, wenn der Gläubiger wegen der $\mathrm{zu}$ vollstreckenden Forderungen insgesamt auf eine in einer Anlage beigefügte Forderungsaufstellung verweist, auch wenn eine zutreffende Eintragung der zu vollstreckenden Forderungen in die vorgegebene Forderungsaufstellung teilweise möglich gewesen wäre.

b) Das Antragsformular bietet auf Seite 3 keine vollständige und zutreffende Eintragungsmöglichkeit, wenn der Gläubiger die Vollstreckung wegen mehrerer Kostenforderungen nebst Zinsen mit gleicher Zinshöhe, aber unterschiedlichen Zinsläufen betreibt.

c) Das Vollstreckungsgericht ist im Rahmen des streng formalisierten Zwangsvollstreckungsverfahrens nicht befugt, eine vom Gläubiger vorgenommene Verrechnung an ihn geleisteter Zahlungen auf ihre Richtigkeit gemäß $\$ 367$ Abs. 1 BGB hin zu überprüfen.

Auch wenn der BGH in allen Entscheidungen die Unterscheidung in Antrag und gesamtes Formular nicht erörtert hat, bin ich weiterhin der Auffassung, dass in erster Linie nur der Antrag verbindlich ist und nicht das gesamte Formular. Für das vorliegende Werk bleibe ich daher bei dem den jeweiligen Formularen vorangestellten Hinweis, entweder den zu pfändenden Anspruch im Feld „G“ einzutragen oder eine gesonderte Anlage zu benutzen.

In den nachfolgenden „Mustern“ wird davon ausgegangen, dass der Antrag in jedem Falle verbindlich ist. Es wird weiter davon ausgegangen, dass auch die Formularfelder vom Antragsteller soweit möglich und rechtlich zulässig genutzt werden müssen. Falls der Antragsteller von dem vorgegebenen Formularinhalt abweichen möchte, muss er dies kenntlich machen, das Vollstreckungsgericht muss dann ggf. den beigefügten Beschlussentwurf bei seiner Entscheidung ändern. Dies gilt insbesondere immer dann, wenn bei den Formularfeldern der vorgegebene Text fehlerhaft, ungenau oder in der Rechtsprechung strittig behandelt wird. Hierauf wird bei den jeweiligen Mustern ausdrücklich hingewiesen.

5. Erläuterungen $\mathrm{zu}$ den einzelnen Mustern sind meist nach gleichbleibendem Schema gegliedert:

Zifferngruppe 0 (Vorbemerkungen) verweist auf vertiefende Darstellung an anderer Stelle oder auf spezielle Muster (s. unter 6.).

Zifferngruppe 1 behandelt Materiell-rechtliches $\mathrm{zu}$ dem zu pfändenden Anspruch oder anderem Recht.

Zifferngruppe 2 behandelt dessen Pfändung und Verwertung.

Zifferngruppe 3 gibt bei Bedarf besondere Hinweise, z.B. zum Rechtsweg oder zur Abgrenzung von ähnlichen Ansprüchen.

6. Die Muster M 12 ff. zeigen den „speziellen Teil“ des Pfändungsantrags bzw. des Pfändungs- und Überweisungsbeschlusses, auch bezüglich etwa angezeigter anderweitiger Verwertung, ohne Rubrum, Forderungsberechnung usw. Dieser „spezielle Teil“ ist entweder identisch mit dem verbindlichen Formulartext oder kann ansonsten in dem amtlichen Vordruck unter „Anspruch G“ oder „gemäß gesonderter An- 
lage“ eingefügt werden. Soweit eine andere Ansicht vertreten wird, als in dem amtlichen Vordruck vorgesehen, wird ausdrücklich darauf hingewiesen.

\section{Hinweise dazu:}

In dem amtlichen Formular lautet der Text zu dem Verbot an den Drittschuldner (Arrestatorium), das Gebot an den Schuldner (Inhibitorium) und die Überweisung: „Der Drittschuldner darf, soweit die Forderung gepfändet ist, an den Schuldner nicht mehr zahlen. Der Schuldner darf insoweit nicht über die Forderung verfügen, sie insbesondere nicht einziehen.

Zugleich wird dem Gläubiger die zuvor bezeichnete Forderung in Höhe des gepfändeten Betrages

- zur Einziehung überwiesen.

- an Zahlungs statt überwiesen."

In den Mustern findet sich wiederholt ein sprachlich angepasster Text, da z.B. bei einem anderen Vermögensrecht der Begriff „Forderung“ nicht passt. Die Notwendigkeit, sich im Pfändungs- und Überweisungsbeschluss exakt und möglichst eindeutig auszudrücken, ist ebenso verpflichtend wie in jedem anderen Bereich auch. Es gilt insbesondere gegenüber dem Drittschuldner und Schuldner klare, unmissverständliche und eindeutige Anweisungen zu geben. Der Inhalt des Beschlusses sollte dies auch mit Hilfe einer korrekt gewählten Sprache erfüllen.

Ist die Vollstreckungsforderung niedriger als die zu pfändende Forderung, so muss zur Vermeidung einer Überpfändung klargestellt werden, dass es sich um eine Teilpfändung handelt, z.B. durch folgende Formulierungen:

Wegen dieser Ansprüche, ... und in Höhe dieser Beträge wird der entsprechende Teil der angeblichen Forderung des Schuldners gegen ... gepfändet, der gepfändete Teil der Forderung hat Vorrang vor dem Rest.

Dies ist in dem amtlichen Vordruck nicht vorgesehen. Der Antragsteller sollte dies in seinem Antrag formulieren und darauf achten, dass das Gericht dies im Beschluss übernommen hat.

7. Beginnen mehrere Zeilen eines Musters mit einem Gedankenstrich, bedeutet das den Hinweis, dass mehrere Alternativen gezeigt sind, unter denen zu wählen ist. 